\title{
9 Conclusion
}

The pure theory of international trade has come a long way from Ricardo's comparative cost doctrine, but we are still a long way from explaining many of the trade phenomena which exist in the world today. Part of the reason for this is that trade theory has remained in the realm of comparative statics with little attention placed on dynamics. This is inevitable in such a complicated interrelated system. Trade theory must explicitly take into account non-economic objectives and place trade theory in a larger socio-politico-economic setting if we wish the theory to be of any practical use. In this sense we would be returning to issues which the classical economists knew were important, but with the difference that our value judgements are made explicit, and with a greater understanding of the economic system. 